\title{
ON DEGREE OF APPROXIMATION ON A JORDAN CURVE TO A FUNCTION ANALYTIC INTERIOR TO THE CURVE BY FUNCTIONS NOT NECESSARILY ANALYTIC INTERIOR TO THE CURVE
}

\author{
J. L. WALSH
}

It is our object here to consider the subject mentioned in the title by proving the following theorem.

ThEOREM. Let $C$ be a rectifiable Jordan curve in the plane of the complex variable $z$, and let the function $f(z)$ be analytic interior to $C$, continuous in the corresponding closed region. Let each of the functions $f_{n}(z), n=1,2, \cdots$, be analytic exterior to $C$ and continuous in the corresponding closed region, except perhaps for poles of total order not greater than $n$ exterior to $C$. We write

$$
f_{n}(z) \equiv g_{n}(z)+h_{n}(z),
$$

for $z$ on and exterior to $C$, where $g_{n}(z)$ is a rational function of $z$ with all its poles exterior to $C$ and $h_{n}(z)$ is analytic in the extended plane exterior to $C$, continuous in the corresponding closed region, with $h_{n}(\infty)=0$. If the totality of poles of all the $f_{n}(z)$ have no limit point on $C$, and if we have

$$
\limsup _{n \rightarrow \infty}\left[\max \left|f(z)-f_{n}(z)\right|, z \text { on } C\right]^{1 / n} \leqq 1 / R<1,
$$

then we have also

$$
\begin{aligned}
& \underset{n \rightarrow \infty}{\limsup }\left[\max \left|f(z)-g_{n}(z)\right|, z \text { on } C\right]^{1 / n} \leqq 1 / R, \\
& \underset{n \rightarrow \infty}{\lim \sup }\left[\max \left|h_{n}(z)\right|, z \text { on } C\right]^{1 / n} \leqq 1 / R .
\end{aligned}
$$

The significance of this theorem is in part as follows. In numerous situations a function $f(z)$ is approximated on a Jordan curve $C$ by a function of type $f_{n}(z)$; for instance $f(z)$ may be approximated on the unit circle $C:|z|=1$ by a trigonometric polynomial in the arc length, of order $n$, which is of type $f_{n}(z)$. If this approximating function $f_{n}(z)$ is split into the two components $g_{n}(z)$ and $h_{n}(z)$, what does $h_{n}(z)$ contribute to the degree of approximation? This question is answered by the theorem, asserting that asymptotically $h_{n}(z)$ contributes nothing to the degree of approximation, insofar as approximation is measured by the first member of (2); it is no more favorable to approximate

Received by the editors December 15, 1945. 
to $f(z)$ on $C$ by functions $f_{n}(z)$ than by their respective components $g_{n}(z)$. As a matter of comparison, we note that if $C$ is the unit circle, each of the functions $h_{n}(z)$ is orthogonal ${ }^{1}$ to $f(z)$ on $C$, and consequently in the sense of least squares no $h_{n}(z)$ can contribute to approximation to $f(z)$ on $C$.

The present problem, approximation by functions not analytic in a given region to a function analytic in that region, is in a sense a companion piece to a problem studied on various occasions ${ }^{2}$ by the present writer, namely approximation by functions analytic in a region to a function not analytic in that region.

The theorem is of course essentially more general than approximation on the unit circle. The theorem is not in variant under arbitrary conformal transformation of either the interior or exterior of $C$; such a transformation does not leave unchanged the components of $f_{n}(z)$ indicated in (1).

In connection with this theorem, it is to be noted ${ }^{8}$ that any function $f(z)$ which satisfies the given hypothesis and is analytic also on $C$ can be uniformly approximated on $C$ so that (2) is satisfied for suitably chosen $R$, by rational functions $f_{n}(z) \equiv g_{n}(z)$ of respective degrees $n$ with poles in $n$ prescribed points exterior to $C$, the prescribed poles having no limit point on $C$.

We proceed to the proof of the theorem. Let $\Gamma_{1}$ and $\Gamma_{2}$ be two analytic Jordan curves chosen with $\Gamma_{2}$ interior to $\Gamma_{1}$, and $C$ interior to $\Gamma_{2}$, and so chosen that all the poles of every $f_{n}(z)$ lie exterior to $\Gamma_{1}$. For $z$ between $\Gamma_{1}$ and $\Gamma_{2}$ we have

$$
f_{n}(z) \equiv \frac{1}{2 \pi i} \int_{\Gamma_{1}} \frac{f_{n}(t) d t}{t-z}+\frac{1}{2 \pi i} \int_{\Gamma_{2}} \frac{f_{n}(t) d t}{t-z},
$$

the integrals being extended in the positive sense with respect to the annular region bounded by $\Gamma_{1}$ and $\Gamma_{2}$. The function

$$
g_{n}(z) \equiv \frac{1}{2 \pi i} \int_{\Gamma_{1}} \frac{f_{n}(t) d t}{t-z}, \quad z \text { interior to } \Gamma_{1},
$$

is defined and analytic throughout the interior of $\Gamma_{1}$, and the function

$$
h_{n}(z) \equiv \frac{1}{2 \pi i} \int_{\Gamma_{2}} \frac{f_{n}(t) d t}{t-z}, \quad z \text { exterior to } \Gamma_{2},
$$

${ }^{1}$ See for instance Walsh, Interpolation and approximation by rational functions in the complex domain, Amer. Math. Soc. Colloquium Publications, vol. 20, New York, $1935, \$ 6.11$.

${ }^{2}$ Loc. cit. $\$ \$ 6.11$ et seq., $\$ \$ 11.3$ et seq.

3 This result is due to Shen; loc. cit. $\$ 9.7$. 
is defined and analytic in the extended plane exterior to $\Gamma_{2}$, with $h_{n}(\infty)=0$. The second members of (5) and (6) for fixed $z$ are independent of continuous deformation of $\Gamma_{1}$ and $\Gamma_{2}$ provided that $\Gamma_{1}$ and $\Gamma_{2}$ in the deformation do not cross $z$ or singularities of $f_{n}(z)$.

The equation

$$
f_{n}(z) \equiv g_{n}(z)+h_{n}(z),
$$

valid for $z$ between $\Gamma_{1}$ and $\Gamma_{2}$, is also valid wherever the functions involved can be defined by analytic extension. It follows from (7) that in the extended plane exterior to $\Gamma_{2}$ the singularities of $g_{n}(z)$ are precisely the singularities of $f_{n}(z)$, namely poles of total order not greater than $n$; moreover $g_{n}(z)$ is analytic interior to $\Gamma_{1}$, hence is a rational function of order not greater than $n$. The function $h_{n}(z)$, defined by (6) and suitably extended analytically by (7), is analytic in the extended plane exterior to $C$, continuous in the corresponding closed region.

The separation of $f_{n}(z)$ into components as defined by (1) is necessarily unique, for if we have in the closed exterior of $C$

$$
g_{n}^{(1)}(z)+h_{n}^{(1)}(z) \equiv g_{n}^{(2)}(z)+h_{n}^{(2)}(z),
$$

where $g_{n}{ }^{(1)}(z)$ and $g_{n}{ }^{(2)}(z)$ satisfy the prescribed conditions for $g_{n}(z)$ and $h_{n}{ }^{(1)}(z)$ and $h_{n}{ }^{(2)}(z)$ satisfy the prescribed conditions for $h_{n}(z)$, then the function $g_{n}{ }^{(1)}(z)-g_{n}{ }^{(2)}(z)$ is rational with its poles (if any) exterior to $C$, but is also equal to $h_{n}{ }^{(1)}(z)-h_{n}{ }^{(2)}(z)$ exterior to $C$, hence is analytic in the entire extended plane, with the relation $g_{n}{ }^{(1)}(\infty)-g_{n}{ }^{(2)}(\infty)=h_{n}{ }^{(2)}(\infty)-h_{n}{ }^{(2)}(\infty)=0$; thus we have $g_{n}{ }^{(1)}(z)$ $\equiv g_{n}{ }^{(2)}(z)$ and $h_{n}{ }^{(1)}(z) \equiv h_{n}{ }^{(2)}(z)$.

We denote generically by $C_{\rho}$ the image in the $z$-plane of the circle $|w|=\rho>1$ under the conformal map of the exterior of $C$ onto the region $|w|>1$, with the points at infinity in the two planes corresponding to each other. Let $R_{1}$ be chosen so that the poles of all the $f_{n}(z)$ exterior to $C$ lie exterior also to $C_{R_{1}}$.

Choose $M$ and $R_{0}, 1<R_{0}<R$, so that for $n=1,2, \cdots$ we have from (2)

$$
\left|f(z)-f_{n}(z)\right| \leqq M / R_{0}^{n}, \quad \quad z \text { on } C \text {; }
$$

we have also

$$
\left|f(z)-f_{n+1}(z)\right| \leqq M / R_{0}^{n+1}, \quad z \text { on } C,
$$

whence

$$
\left|f_{n}(z)-f_{n+1}(z)\right| \leqq 2 M / R_{0}^{n}, \quad z \text { on } C \text {, }
$$


where $f_{n}(z)-f_{n+1}(z)$ has at most a total of $2 n+1$ poles exterior to $C$; all such poles lie exterior to $C_{R_{1}}$. By consideration of the function $f_{n}(z)-f_{n+1}(z)$ under the conformal map of the exterior of $C$ in the $z$-plane onto the exterior of the unit circle in the $w$-plane, we have ${ }^{4}$

$$
\left|f_{n}(z)-f_{n+1}(z)\right| \leqq \frac{2 M}{R_{0}^{n}}\left(\frac{R_{1} \rho-1}{R_{1}-\rho}\right)^{2 n+1} \text { for } 1<|w|=\rho<R_{1} .
$$

Choose $\rho$ so near unity that we have

$$
\frac{1}{R_{0}}\left(\frac{R_{1} \rho-1}{R_{1}-\rho}\right)^{2}<1 .
$$

It then follows from (8) that the sequence $f_{n}(z)$ converges uniformly on $C_{p}$. By (2) the sequence $f_{n}(z)$ converges to $f(z)$ uniformly on $C$, hence converges uniformly in the closed annulus bounded by $C$ and $C_{\rho}$, to a limit which in the annulus we now denote by $f(z)$. It is a consequence of the rectifiability of $C$ that the function $f(z)$ as just enlarged in definition is analytic also on $C$ itself, hence analytic throughout the interior of $C_{\rho}$. By virtue of (8) and (9) we can write

$$
\left|f(z)-f_{n}(z)\right| \leqq \frac{M_{1}}{R_{0}^{n}}\left(\frac{R_{1} \rho-1}{R_{1}-\rho}\right)^{2 n}, \quad z \text { on } C_{\rho},
$$

where $M_{1}$ depending on $\rho$ is suitably chosen.

If we now set

$$
f(z)=\frac{1}{2 \pi i} \int_{C_{\rho}} \frac{f(t) d t}{t-z}, \quad z \text { interior to } C_{\rho},
$$

and also choose $\Gamma_{1}$ in (5) as $C_{\rho}$, inequality (3) follows from (5) and (11) by virtue of (10); it will be noted that (10) implies

$$
\limsup _{n \rightarrow \infty}\left[\max \left|f(z)-g_{n}(z)\right|, z \text { on } C\right]^{1 / n} \leqq \frac{1}{R_{0}}\left(\frac{R_{1} \rho-1}{R_{1}-\rho}\right)^{2},
$$

and in the second member of (12) we can allow $R_{0}$ to approach $R$ and $\rho$ to approach unity.

Inequality (4) is a consequence of inequalities (2) and (3), by virtue of (1). The theorem is established.

CoROLLARY. The theorem remains valid if $C$ is not assumed rectifiable, but if $f(z)$ is assumed analytic in the closed interior of $C$.

${ }^{4}$ Loc. cit. p. 231, Lemma I. The requirement in this Lemma I that $P(z)$ be rational may be replaced by the requirement that $P(z)$ be continuous on $|z|=r$, analytic in $r<|z|<\rho r$, meromorphic with not more than $n$ poles in $|z| \geqq \rho r$. 
The proof of the corollary follows directly the proof of the theorem; in (9) we choose $\rho$ so near unity that $f(z)$ is analytic on and within $C_{\rho}$; the identity in the annulus bounded by $C$ and $C_{\rho}$ of the given function $f(z)$ and the limit of the sequence $f_{n}(z)$ follows from the fact that these two functions analytic in the annulus approach the same set of boundary values when $z$ exterior to $C$ approaches $C$.

Theorem and corollary can be extended to include point sets $C$ much more general than Jordan curves, even the case where $C$ consists of several disjoint continua; these extensions are left to the reader.

The theorem becomes false if the requirement is omitted that $C$ be rectifiable and if no additional requirement is placed on $f(z)$. For under such conditions $C$ may be chosen (Osgood) to have positive area; there exists (Pompeiu) a function $f(z)$ analytic in the extended plane except on $C$, continuous for all values of $z$, zero at infinity yet not identically zero. We can choose $f_{n}(z) \equiv f(z), n=1,2, \cdots$, from which it follows that (2) is satisfied for arbitrary $R$. Yet we have $g_{n}(z) \equiv 0, h_{n}(z) \equiv f_{n}(z)=f(z)$, so that the first member of both (3) and (4) can be written

$$
\limsup _{n \rightarrow \infty}[\max |f(z)|, z \text { on } C]^{1 / n},
$$

which has the value unity. Inequalities (3) and (4) of the conclusion of the theorem are not satisfied.

HARVARD UNIVERSITY 